\title{
LIDERAZGO DIRECTIVO Y DESEMPEÑO DOCENTE EN UNA INSTITUCIÓN EDUCATIVA PARROQUIAL DEL DISTRITO DE SAN ISIDRO'
}

\author{
Mg. Laura Morayma Arana Agûero \\ Mg. Janette María Coronado Tarrillo
}

\section{RESUMEN}

La investigación tuvo como propósito determinar la relación entre los estilos de liderazgo directivo y el desempeño docente en una Institución Educativa Parroquial del distrito de San Isidro. El diseño utilizado fue correlacional y el instrumento empleado fue el Cuestionario de liderazgo directivo elaborado por Paz H. y Zamora C. (2014). Para efectos de la investigación se aplicó a toda la población, 48 docentes. Para medir el desempeño docente se tomaron en cuenta las evaluaciones que fueron obtenidas de la direccion de la institución. Los resultados demostraron que existe una relación directa y significativa entre estas dos variables, señalando que el estilo que más predomina es el Liderazgo Transformacional y un alto nivel de desempeño de los docentes en la institución educativa.

Palabras clave: Liderazgo directivo, desempeño docente

\begin{abstract}
The research aims to determine the relationship between the styles of management leadership and teacher performance at school in San Isidro. The design it was used the correlacional and the instrument it was used the leadership directive questionnaire and this was made by Paz H. and Zamora C. (2014). It was applied to all the 48 teachers of the school. In order to measure the teacher performance were taken into account the evaluations that were obtained from the Academic Management department of the institution. The results showed that there is a direct and significant relationship between these two variables, point out that the most predominant style is the Transformational Leadership, and there is a high level of performance of teachers of the school.
\end{abstract}

Keywords: executive leadership and teaching performance.

1 Tesis de investigación para obtener el grado de Maestro 


\section{INTRODUCCIÓN}

Actualmente, el liderazgo es un tema que ha sido estudiado ampliamente por diversos autores, quienes han aportado desde los diferentes enfoques, múltiples conceptos que han ayudado a consolidar el presente trabajo de investigación. Su estudio se ha convertido en uno de los principales argumentos de la literatura empresarial y en una de las mayores preocupaciones de las organizaciones educativas.

Es importante y propicio resaltar que a nivel nacional e internacional se está priorizando la mejora del sector educativo, implicando a los líderes como principales gestores de los logros y deficiencias que usualmente suceden en dicho sector. "Los gobiernos de todo el mundo están dedicando más recursos que nunca al desarrollo de líderes en las escuelas. Miembros del mundo de los negocios, siempre entusiasmados con el romance del liderazgo, piensan que los problemas de los colegios coinciden con deficiencias en sus líderes" (Mulford, 2006, p.2).

Las deficiencias del liderazgo son vividas dentro de cualquier organización educativa y esto repercute considerablemente en el desempeño de los docentes. Sin embargo, para lograr una buena gestión en una organización educativa es necesario extender la mirada y obtener una visión holística que posibilite saber los reales alcances de la labor de los directivos de una organización educativa. Es, por tanto, un proceso ciertamente complejo, que demanda a su vez reorientar la labor educativa hacia lo que se denomina en la actualidad el liderazgo y todo lo que ello implica.

En consecuencia, el liderazgo es el punto de partida para lograr una buena gestión; cada líder expresará un estilo de liderazgo, fuente de poder y diversas ideologías. "El estilo de liderazgo se refiere al patrón típico de conducta que adopta el líder o el directivo con el propósito de guiar a sus trabajadores para que alcancen las metas de la organización. El estilo está basado en experiencias, el género, la educación y la capacitación, y es el estímulo que motiva al líder en diferentes circunstancias" (Madrigal, 2005, p. 76).

Dentro de un contexto educativo es fundamental e indispensable tener un líder que cuente con un estilo que realice y alcance las metas dentro de su propuesta educativa. En consecuencia, el líder es la persona que tiene características y cualidades que los demás no poseen en alguna dimensión de la actividad humana, profesional o personal. Estas son las cualidades que debe poseer un líder.

Por tal razón, la presente investigación tiene como propósito principal determinar la relación entre los estilos de liderazgo directivo y el desempeño docente en una Institución Educativa Parroquial del distrito de San Isidro. 
Se considera que, a partir de los resultados de esta investigación, se logrará identificar que existe una relación significativa entre el estilo de liderazgo predominante del directivo y el desempeño de los docentes.

\section{BASES TEÓRICAS}

\section{El liderazgo}

\section{Definición}

El liderazgo es un tema que ha sido estudiado por muchos autores, quienes han aportado desde los diferentes enfoques, múltiples conceptos. Al respecto Madrigal sostiene:

Liderazgo es la acción de influir en los demás; actitudes, conductas y habilidades de dirigir, orientar, motivar, vincular, integrar y optimizar el quehacer de las personas y grupos para lograr los objetivos deseados, en virtud de su posición en la estructura de poder y promover el desarrollo de sus integrantes (2011, p. 18).

En consecuencia, el liderazgo es el punto de partida para lograr una buena gestión. Cada líder expresará un estilo de liderazgo, fuente de poder y diversas ideologías. Al respecto se señala que:

El estilo de liderazgo se refiere al patrón típico de conducta que adopta el líder o el directivo con el propósito de guiar a sus trabajadores para que alcancen las metas de la organización. El estilo está basado en experiencias, el género, la educación y la capacitación, y es el estímulo que motiva al líder en diferentes circunstancias (Madrigal, 2005, p.76).

El liderazgo, como proceso de dirigir las actividades laborales de los miembros de un grupo y de influir en ellas, tiene algunas implicaciones importantes:

- El liderazgo involucra a otras personas: a los empleados o seguidores. Los miembros del grupo, dada su voluntad para aceptar las órdenes del líder, ayudan a definir la posición del líder y permiten que transcurra el proceso del liderazgo; si no hubiera a quien mandar, las cualidades del liderazgo serían irrelevantes.

- El liderazgo entraña una distribución desigual del poder entre los líderes y los miembros del grupo. Los miembros del grupo no carecen de poder; pueden dar forma, y de hecho 
lo hacen, a las actividades del grupo de distintas maneras. Sin embargo, por regla general, el líder tendrá más poder.

- El liderazgo es la capacidad para usar las diferentes formas del poder para influir en la conducta de los seguidores, de diferentes maneras. De hecho algunos líderes han influido en los soldados para que mataran y algunos líderes han influido en los empleados para que hicieran sacrificios personales para provecho de la compañía.

\section{LIDERAZGO EN LA ACTUALIDAD}

Actualmente el tema del liderazgo encierra diversos y complejos conceptos y la búsqueda de este concepto se ha convertido en uno de los principales argumentos de la literatura empresarial y en una de las mayores preocupaciones de las organizaciones educativas. En la naturaleza de las organizaciones educativas se suele entender por liderazgo cualquier intento que pretenda influir sobre la conducta de otro individuo o grupo, dentro de los cuales podríamos resaltar que para liderar una organización o institución se necesita de habilidades y capacidades (Trechera, 2004).

Entre las destrezas que un líder tendrá que poseer serán las habilidades directivas como el autoconocimiento, el autocontrol, la motivación y especialmente la habilidad de entender las señales emotivas y conductuales de los demás: "empatía" (Whetten y Cameron, 2005).

El liderazgo determina el camino de una organización; es entonces de suma importancia saber que además de las habilidades directivas el líder sea capaz no sólo de conocer lo correcto sino de hacer lo correcto; de lo contrario, si conoce lo correcto y no puede lograrlo, fracasa por ineficaz. Dentro de un contexto educativo es fundamental e indispensable tener un líder con dichas características para que lleve la dirección a realizar y alcanzar las metas dentro de su propuesta educativa. En consecuencia, el líder es la persona que tiene características y cualidades que los demás no poseen en alguna dimensión de la actividad humana, profesional o personal. Estas son las cualidades que debe poseer un líder.

\section{PLANTEAMIENTO DEL PROBLEMA}

\subsection{Descripción del problema}

Actualmente el tema del liderazgo ha tomado una gran importancia; no sólo se ha convertido en uno de los principales argumentos de la literatura empresarial, sino en una de las mayores preocupaciones de las organizaciones educativas.

La educación actual exige cambios significativos, como es la búsqueda del líder directivo, lo cual puede parecer un ideal, considerando que los seres humanos son 
tan complejos y diferentes, que resulta difícil encontrar a ese directivo: "se necesita directores líderes que armonicen adecuadamente los factores de calidad con los procesos de aprendizaje y sean movilizadores del cambio; quienes no se esfuercen por cambiar su estructura mental, jamás podrán optimizar sus logros de gestión y acción educativa" (Alfonso, 2001, p.13).

En las instituciones educativas existe hoy un gran interés por el liderazgo, ya que es una necesidad el conducir eficaz y eficientemente los centros educativos, para lograr que sus colaboradores se comprometan con el logro de los objetivos comunes de la institución. Al respecto Chiavenato (2009) sostiene:

Liderazgo es la influencia interpersonal ejercida en una situación, dirigida a través del proceso de comunicación humana a la consecución de uno o diversos objetivos específicos (p.18).

Por ello el liderazgo es un aspecto central en el modelo de gestión educativa y el motor de cualquier organización. Sin embargo, el problema de las organizaciones de educación en nuestro país es el de adolecer de líderes directivos que cuenten con características que los lleven a realizar y alcanzar sus metas. De este modo, se considera que el liderazgo es importante porque no todos los directivos asumen un liderazgo de relevancia que oriente, lidere y dirija a sus colaboradores, sino por el contrario se preocupan más en los resultados que en los propios docentes.

El desempeño docente es otro elemento clave para impedir o promover cambios en las instituciones educativas, siempre y cuando existan las condiciones necesarias, que incrementen la comunicación, la calidad del trabajo y el compromiso para que los aprendizajes puedan darse de la mejor manera posible, en función de que los alumnos aprendan, para garantizar la calidad total en la institución. Por eso es importante que los líderes no sólo motiven a sus colaboradores a satisfacer sus propias metas y necesidades personales, sino también en la realización de los objetivos deseados para la organización. Referente a los docentes, la UNESCO (2004) señala que los procesos vividos por la mayoría de países latinoamericanos, así como los resultados de estudios diversos, muestran que el factor docente es uno de los más importantes para que las reformas educativas tengan buenos resultados en el aprendizaje de los estudiantes y en la manera como se gestiona la educación en los sistemas, las escuelas y las aulas. Si por un lado, sin buenos docentes no hay cambio posible, por otro lado la situación profesional y social de los docentes es uno de los nudos críticos de la educación. Esta es una causa importante del problema, pero también puede ser el inicio de las estrategias más efectivas para transformar la educación y dar una educación de calidad. 
En conclusión, determinar la relación que existe entre el liderazgo directivo y el desempeño docente de una Institución Educativa Parroquial del distrito de San Isidro, nos permitirá conocer los estilos de liderazgo predominantes de los directivos de la institución y establecer su influencia en el desempeño docente.

\subsection{Formulación del problema}

Problema general

¿Qué relación existe entre los estilos de liderazgo directivo y el desempeño docente en la Institución Educativa Parroquial del distrito de San Isidro?

\section{Problemas específicos}

1.- ¿Cuáles son los estilos de liderazgo directivo en la Institución Educativa Parroquial del distrito de San Isidro?

2.- ¿Cuáles son los niveles de desempeño docente en la Institución Educativa Parroquial del distrito de San Isidro según niveles educativos?

3. ¿Qué relación existe entre los estilos de liderazgo directivo y el desempeño docente en el nivel de educación inicial de la Institución Educativa Parroquial del distrito de San Isidro?

4.- ¿Qué relación existe entre los estilos de liderazgo directivo y el desempeño docente en el nivel de educación primaria de la Institución Educativa Parroquial del distrito de San Isidro?

5.- ¿Qué relación existe entre los estilos de liderazgo directivo y el desempeño docente en el nivel de educación secundario de la Institución Educativa Parroquial del distrito de San Isidro?

\subsection{Justificación}

Desde el punto de vista práctico la presente investigación analizará la labor de la autoridad, del director, como líder y su incidencia en el desempeño docente de los educadores en la Institución Educativa Parroquial del distrito de San Isidro y a la vez permitirá al directivo conocer sus estilos de liderazgo según la percepción que tienen los docentes de su gestión. Esto podrá contribuir a la gestión y calidad educativa de la institución, así como posibles alternativas de solución, de tal manera que pueda lograr los desafíos de la educación que se están trazando en la actualidad. 
Muchas instituciones educativas buscan responder a las demandas de la sociedad de este mundo globalizado caracterizado por una alta competitividad y la búsqueda de la excelencia de la calidad de los servicios que ofrecen tanto los individuos como las organizaciones.

Las autoridades de las instituciones educativas no pueden estar al margen de las grandes transformaciones y expectativas que se están dando. Para que estas organizaciones puedan estar bien manejadas y así lograr una mejor calidad educativa, deben contar con recursos humanos de óptima preparación, adecuados para ocupar los puestos directrices en los distintos niveles.

Además, las instituciones educativas tienen un rol fundamental en la formación integral de los estudiantes. Esa responsabilidad, en gran parte es asumida por los docentes, quienes a diario trabajan con ellos. Por lo tanto, el liderazgo directivo y el desempeño docente deben ser evaluados y analizados, lo cual permitirá conocer los estilos de liderazgo predominantes de los directivos de la institución y establecer su influencia en el desempeño docente en diferentes aspectos.

Por eso el director es la pieza clave en la institución educativa. El éxito en el cumplimiento de las finalidades institucionales depende en gran medida de su capacidad de idear, conducir y movilizar a la comunidad educativa hacia los objetivos y metas que más convengan a la organización. Además, el aprovechamiento del potencial que tiene cada profesor y el equipo docente en general están fuertemente condicionados por la capacidad del director de estimularlos a trazarse metas crecientemente ambiciosas.

Estos resultados permitirán contrastar los aspectos de los estilos de liderazgo directivo y el desempeño docente como importantes factores vinculados al cambio y mejora de la calidad educativa. Al hacerse evidente que los actores de la enseñanza son influenciados en su labor por diversos elementos, se precisará el conocer si hay una relación significativa entre el tipo de liderazgo directivo y el desempeño docente de la institución educativa.

\section{OBJETIVOS}

\section{General}

Determinar la relación de los estilos de liderazgo directivo y el desempeño docente en la Institución Educativa Parroquial del distrito de San Isidro.

\section{Específicos}

1. Describir los estilos de liderazgo directivo en la Institución Educativa Parroquial del distrito de San Isidro. 
2. Describir los niveles de desempeño docente en la Institución Educativa Parroquial del distrito de San Isidro según niveles educativos.

3. Establecer la relación entre los estilos de liderazgo directivo y el desempeño docente en el nivel de educación inicial de la Institución Educativa Parroquial del distrito de San Isidro.

4. Establecer la relación entre los estilos de liderazgo directivo y el desempeño docente en el nivel de educación primaria de la Institución Educativa Parroquial del distrito de San Isidro.

5. Establecer la relación entre los estilos de liderazgo directivo y el desempeño docente en el nivel de educación secundaria de la Institución Educativa Parroquial del distrito de San Isidro.

\section{METODOLOGÍA}

\section{Tipo de investigación}

Es cuantitativa, porque emplea procesos metódicos y empíricos para generar conocimientos. De las preguntas se establecieron hipótesis y se determinaron variables, trazándose un plan para probarlas; se miden las variables en un contexto determinado, se analizan las mediciones utilizándose métodos estadísticos y extrayendo conclusiones respecto a las hipótesis. (Hernández, Fernández \& Baptista 2014).

\section{Diseño de investigación}

Es una investigación con un diseño no experimental, transaccional y correlacional. Este diseño busca describir relaciones entre dos o más categorías y los datos son recolectados en un solo momento, en un tiempo único. (Hernández, Fernández y Baptista 2014). En este sentido se buscó medir la relación entre los estilos de liderazgo directivo y el desempeño docente en una Institución Educativa Parroquial del distrito de San Isidro.

\section{Variables}

\section{Variables que se relacionan:}

Liderazgo directivo

Desempeño docente 


\section{Definición de variables}

\section{Liderazgo}

Chiavenato (2009). "Liderazgo es la influencia interpersonal ejercida en una situación, dirigida a través del proceso de comunicación humana a la consecución de uno o diversos objetivos específicos".

Madrigal (2011). "Liderazgo es la acción de influir en los demás; actitudes conductas y habilidades de dirigir, orientar, motivar, vincular, integrar y optimizar el quehacer de las personas y grupos para lograr los objetivos deseados, en virtud de su posición en la estructura de poder y promover el desarrollo de sus integrantes".

\section{Desempeño docente}

"Proceso sistemático mediante el cual se evalúa la habilidad del docente, su potencial de desarrollo futuro y su rendimiento". (Saravia, 2005).

"El desempeño es una apreciación sistemática de cada persona con respecto a un cargo o empleo que potencialmente debe desarrollarse en el futuro. Se puede decir que es un medio que permite resolver problemas, por lo que requiere de supervisión de personal, integración del empleado a la organización o al cargo que ocupa en la actualidad, solventar desacuerdos, aprovechamiento del potencial para un cargo, motivación entre otros" (Chiavenato, 2006).

\section{Definición operacional}

\section{Estilos de liderazgo directivo}

Definido por los resultados obtenidos al aplicar el Cuestionario de liderazgo directivo (Paz y Zamora, 2014).

\section{Desempeño docente}

Definido por los resultados obtenidos de fuentes secundarias después de aplicarles la evaluación docente. Información proporcionada por la Dirección de la institución educativa (2015). 


\section{POBLACIÓN}

\section{Población}

La población de la presente investigación estuvo constituida por 48 docentes de la Institución Educativa Parroquial del distrito de San Isidro, de los cuales el $45.83 \%$ son del nivel de educación inicial, el $29.17 \%$ del nivel de educación primaria y el $25 \%$ corresponden al nivel de educación secundaria, siendo docentes del género femenino $(85.42 \%)$ y docentes de género masculino (14.58\%), clasificados en un rango de edades de 20 a 70 años.

Para efectos de la investigación se trabajó con todos los sujetos de la población, ya que en la institución sólo laboran 48 docentes en los tres niveles educativos.

\section{Muestra}

Para efectos de la investigación no se obtuvo muestra debido a que se trabajó con todos los sujetos de la población, cuyo número es de 48 docentes en los tres niveles educativos.

En la tabla 1 se aprecia que la población de estudio está conformada por 48 docentes de los cuales el $45.83 \%$ corresponden al nivel de educación inicial, el 29.17\% corresponden al nivel de educación primaria y el 25\% corresponden al nivel de educación secundaria.

\section{Tabla 1}

Distribución de los docentes según nivel educativo

\begin{tabular}{c|c|c}
\multirow{2}{*}{ Niveles } & \multicolumn{2}{|c}{ Población } \\
\cline { 2 - 3 } & $\mathbf{N}^{\circ}$ & $\%$ \\
\hline Inicial & 22 & 45.83 \\
\hline Primaria & 14 & 29.17 \\
\hline Secundaria & 12 & 25.00 \\
\hline Total & 48 & 100.00 \\
\hline
\end{tabular}

\section{INSTRUMENTOS}

Para medir la variable Liderazgo Directivo se utilizó el Cuestionario de liderazgo directivo (Anexo A). Este instrumento fue elaborado por las profesoras Paz y Zamora (2014) en la Tesis "Estilos de liderazgo directivo y su relación con la satisfacción laboral docente en dos instituciones educativas privadas de Lima Metropolitana" en el año 2014, para optar 
el grado académico de Maestro en Educación en la Universidad Marcelino Champagnat.

Para el cuestionario de estilos de liderazgo directivo se han considerado las siguientes dimensiones:

- Democrático, habiendo considerado los siguientes indicadores:

- Capacidad de escucha, con un ítem ( $\left.N^{0} 1\right)$

- Participación, con un ítem ( $\left.N^{0} 2\right)$

- Toma de decisiones, con un ítem (No 3)

- Motivación, con un ítem ( $\left.{ }^{0} 4\right)$

- Autocrático, habiendo considerado los siguientes indicadores:

- Dirección, con un ítem ( $\left.N^{0} 5\right)$

- Toma de decisiones, con un ítem ( $\left.\mathrm{N}^{\circ} 6\right)$

- Participación, con un ítem (No 7)

- Obediencia, con un ítem ( $\left.N^{\circ} 8\right)$

- Transformacional, habiendo considerado los siguientes indicadores:

- Carisma o influencia idealizada, con dos ítems ( No 9 y 10)

- Inspiración, con dos ítems (No 11 y 12)

- Estimulación intelectual, con dos ítems (No 13 y 14)

- Consideración individualizada, con dos ítems ( No 15 y 16)

- Estratégico, habiendo considerado los siguientes indicadores:

- Pensamiento estratégico, con dos ítems ( $N^{0} 17$ y 18)

- Actitud estratégica, con dos ítems (No 19 y 20)

- Intención estratégica, con dos ítems ( $N^{0} 21$ y 22)

- Evaluación estratégica, con dos ítems (No 23 y 24)

Los ítems de este cuestionario son de escala tipo Likert, según Hernández, Fernández y Baptista (2014), consiste en un conjunto de ítems presentados en forma de afirmaciones o juicios ante los cuales se pide la reacción de los sujetos a los que se les administra. Para lo cual, las escalas del instrumento Estilos de Liderazgo Directivo, están expresadas en forma 
positiva: "Siempre", "Casi siempre", "A veces", "Nunca", y con sus valores del 1 al 4.

Para medir la segunda variable, Desempeño docente, se tomaron en cuenta las evaluaciones de los docentes como datos secundarios, los que fueron obtenidos del Dpto. de Dirección Académica (Anexo B). Se tuvieron en cuenta los siguientes indicadores.

\section{COMPETENCIAS CARDINALES}

- Compromiso Idente

- Excelencia en el servicio/atención al cliente

- Iniciativa

- Orientación al logro

- Relaciones interpersonales

- Trabajo en equipo

- Adaptación al cambio

- Aprendizaje continuo

- Solución de problemas

- Comunicación

- Planificación y organización

- Técnico-profesionales

\section{COMPETENCIA ESPECÍFICA}

- Liderazgo

- Gestión

- Estilo carismático Idente

\section{RESULTADOS}

6.1. Análisis de la variable liderazgo directivo

El estilo democrático cuenta con cuatro ítems, con una puntuación mínima de 8 y máxima de 16; el estilo autocrático, también de cuatro ítems tiene una puntuación mínima de 10 y máxima de 16. Este segundo estilo presenta un promedio ligeramente mayor y con un valor de dispersión menor al primer estilo. 
El estilo transformacional cuenta con ocho ítems, su valor mínimo es de 18 y el máximo de 32; el estilo estratégico cuenta con la misma cantidad de ítems pero con puntuaciones entre 15 y 32. El primer estilo presenta un promedio ligeramente mayor pero con una dispersión menor que el segundo.

A continuación, en la Tabla 7, se muestra el análisis descriptivo de la variable Estilos de liderazgo directivo.

\section{Tabla 7}

Estadísticos descriptivos de los estilos de liderazgo directivo

\begin{tabular}{c|c|c|c|c|c} 
& M & Mdn & SD & Mínimo & Máximo \\
\hline Democrático & 12.69 & 13 & 2.362 & 8 & 16 \\
\hline Autocrático & 12.71 & 13 & 1.288 & 10 & 16 \\
\hline Transformacional & 25.75 & 26 & 3.867 & 18 & 32 \\
\hline Estratégico & 25.69 & 25.50 & 4.420 & 15 & 32 \\
\hline
\end{tabular}

En la tabla 8 se aprecia que la población de estudio está conformada por 48 docentes de los cuales el $52.08 \%$ corresponden al estilo transformacional, el $47.92 \%$ corresponden al estilo estratégico y el estilo democrático y autocrático con $0 \%$.

\section{Tabla 8}

Distribución de los docentes según estilos de liderazgo

\begin{tabular}{l|c|c}
\multirow{2}{*}{ Estilos } & \multicolumn{2}{|c}{ Docentes } \\
\cline { 2 - 3 } & $\mathbf{N}^{\circ}$ & $\%$ \\
\hline Democrático & 0 & 0 \\
\hline Autocrático & 0 & 0 \\
\hline Transformacional & 25 & 52.08 \\
\hline Estratégico & 23 & 47.92 \\
\hline Total & 48 & 100 \\
\hline
\end{tabular}




\subsection{Análisis de la variable desempeño docente}

Los datos de la variable desempeño docente presenta puntajes entre 38 y 48. Cabe resaltar que la puntuación va en un intervalo de 1 a 50 (1 - 12.5: bajo, 12.5 - 37.5: promedio y 37.5 - 50: alto), por lo que los participantes obtuvieron calificaciones altas.

La media y desviación estándar fue de 43.94 y 2.356, respectivamente y la mediana fue 44. En la Tabla 9 se presentan los resultados del análisis.

\section{Tabla 9}

Resultados estadísticos de la variable desempeño docente

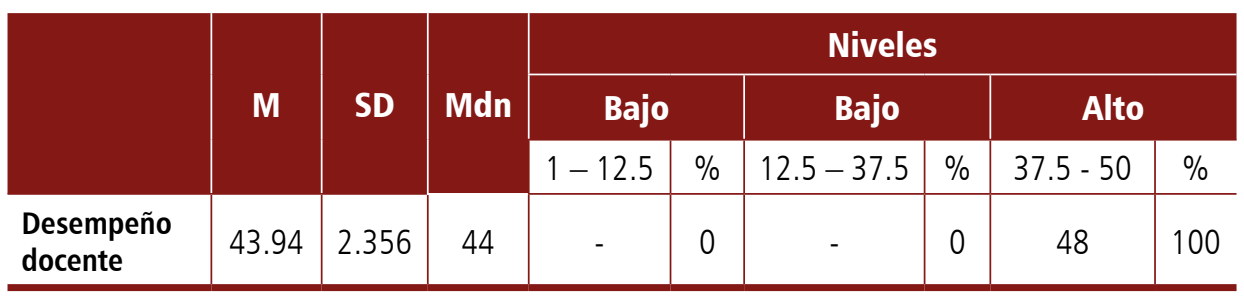

\subsection{Análisis de normalidad}

Con el propósito de realizar la correlación entre variables se utilizó primero la prueba de Shapiro Wilk para observar la distribución de las mismas. En ese sentido, en la Tabla 10 se presentan las pruebas de normalidad para las variables de estudio, en la cual se observa que solo los estilos transformacional y estratégico (ambas de liderazgo directivo) y la variable desempeño docente presentan distribución normal.

Tabla 10

Pruebas de normalidad de las variables de estudio mediante Shapiro Wilk

\begin{tabular}{c|c|c|c|c}
\hline $\begin{array}{c}\text { Variables } \\
\begin{array}{c}\text { Liderazgo } \\
\text { directivo }\end{array}\end{array}$ & $\mathbf{M}$ & Shapiro Wilk & $\mathbf{p}$ \\
\hline Democrático & 12.69 & 2.362 & .936 & .012 \\
\hline Autocrático & 12.71 & 1.288 & .935 & .011 \\
\hline Transformacional & 25.75 & 3.867 & $.960^{*}$ & .105 \\
\hline Transformacional & 25.69 & 4.420 & $.953^{*}$ & .054 \\
\hline Estratégico & 43.94 & 2.356 & $.957^{*}$ & .074 \\
\hline
\end{tabular}

${ }^{*} p<.05$ 
6.4 Análisis de las relaciones entre las variables de estudio: estilos de liderazgo directivo y desempeño docente

Las pruebas estadísticas para el análisis de las correlaciones fueron Pearson, para relacionar los estilos transformacional y estratégico con la variable desempeño docente, y Spearman para relacionar democrático y autocrático con desempeño docente.

A continuación, en la Tabla 11 se presenta las correlaciones entre los estilos de liderazgo directivo y desempeño docente, en donde se observa que los estilos democrático y transformacional tienen coeficientes de correlación directa y significativos de 0.346 y 0.393 ( $p<0.05$ ), por lo que existe relación entre estas dos dimensiones y desempeño docente.

\section{Tabla 11}

Coeficientes de correlación entre estilos de liderazgo directivo y desempeño docente

\begin{tabular}{l|c|c}
\multirow{2}{*}{} & \multicolumn{2}{|c}{ Desempeño docente } \\
\cline { 2 - 3 } & $\mathbf{r}$ & $\mathbf{p}$ \\
\hline Democrático & $.346^{*}$ & .016 \\
\hline Autocrático & .095 & .520 \\
\hline Transformacional & $.393^{*}$ & .006 \\
\hline Estratégico & .260 & .075 \\
\hline
\end{tabular}

$* p<.05$

\section{Relación entre estilos de liderazgo directivo y desempeño docente según nivel de especialidad}

Inicial

Se aplicó las pruebas estadísticas Pearson y Spearman según el análisis anterior.

En este nivel se determinó que solo hay relación entre el estilo transformacional y desempeño docente, puesto que, se obtuvo un coeficiente de correlación directa y significativa de 0.487 ( $<<0.05$ ). En la Tabla 12 se muestran los resultados del análisis de las correlaciones. 


\section{Tabla 12}

Coeficientes de correlación entre estilos de liderazgo directivo y desempeño docente en el nivel de educación inicial

\begin{tabular}{l|c|c}
\multirow{2}{*}{} & \multicolumn{2}{|c}{ Desempeño docente } \\
\cline { 2 - 3 } & $\mathbf{r}$ & $\mathbf{p}$ \\
\hline Democrático & .357 & .102 \\
\hline Autocrático & -.175 & .435 \\
\hline Transformacional & $.487^{\star}$ & .022 \\
\hline Estratégico & .306 & .166 \\
\hline
\end{tabular}

${ }^{*} p<.05$

Primaria

Nuevamente se aplicaron las pruebas de correlación Pearson y Spearman según sea el caso.

En lo que respecta a las correlaciones entre las variables en este nivel (que se presentan en la Tabla 13), solo el estilo transformacional se relaciona con desempeño docente ya que tiene un coeficiente de correlación directa y significativa de 0.470 ( $p<0.05$ ).

\section{Tabla 13}

Coeficientes de correlación entre estilos de liderazgo directivo y desempeño docente en el nivel de educación primario

\begin{tabular}{l|c|c}
\multirow{2}{*}{ Democrático } & \multicolumn{2}{|c}{ Desempeño docente } \\
\cline { 2 - 3 } & $\mathbf{r}$ & $\mathbf{p}$ \\
\hline Autocrático & .381 & .179 \\
\hline Transformacional & .401 & .156 \\
\hline Estratégico & $.470^{\star}$ & .090 \\
\hline
\end{tabular}

${ }^{*} p<.05$ 


\section{Secundaria}

En este nivel, como se observa en la Tabla 14, ningún estilo de liderazgo directivo se relaciona con la variable desempeño docente, ya que las correlaciones no son significativas.

\section{Tabla 14}

Coeficientes de correlación entre estilos de liderazgo directivo y desempeño docente en el nivel de educación secundario

\begin{tabular}{l|c|c}
\multirow{2}{*}{} & \multicolumn{2}{|c}{ Desempeño docente } \\
\cline { 2 - 3 } & $\mathbf{r}$ & $\mathbf{p}$ \\
\hline Democrático & .539 & .071 \\
\hline Autocrático & .417 & .178 \\
\hline Transformacional & .290 & .328 \\
\hline Estratégico & .360 & .298 \\
\hline
\end{tabular}

\section{DISCUSIÓN DE RESULTADOS}

Con respecto a la hipótesis que señala que existe una relación directa y significativa entre los estilos de Liderazgo Directivo y el Desempeño Docente, se observa que los estilos transformacional y estratégico tienen coeficientes de correlación directa y significativos, por lo que existe relación entre estas dos dimensiones y desempeño docente.

Sobresale el liderazgo directivo transformacional, porque el director es un líder que conduce a los docentes a una visión compartida, basado en un proyecto pedagógico que promueven hace 70 años.

Se puede deducir que los directores con este estilo de liderazgo son agentes de cambios, delegan responsabilidades, dan autonomía al trabajo docente y hacen un acompañamiento a su labor.

Estos datos coinciden con los resultados encontrados por Medina (2010) quien halló un estilo predominante de tipo transformacional. En su muestra de estudio, la mayoría de los docentes percibieron un acompañamiento del director líder en el desarrollo de sus capacidades individuales; además, el estilo transformacional de su gestión promovió que los docentes, a su vez, sean líderes con los colegas nuevos que se integraban a la institución. 
También señala que el líder transformador permite la confluencia en las relaciones interpersonales, propicia un acompañamiento y una mayor disposición al aprendizaje cuando los docentes se dejan guiar y orientar por otros docentes con mayores conocimientos y experiencia, que los acogen y los acompañan en su proceso de inserción y adaptación a la cultura organizacional del centro educativo.

Del mismo modo, estos hallazgos se pueden explicar teniendo en cuenta que el líder transformacional es capaz de expresar su visión a los demás, de tal manera que los comprometa y que por lo tanto las actividades diarias se vean imbuidas por sus significados y valores. Busca también que la visión se sostenga o institucionalice en la cultura de la institución educativa. (Burns, citado por Beare, Caldwell y Millikan. 1992).

Con respecto a la hipótesis principal que señala que existe una relación directa y significativa entre los estilos de liderazgo directivo y el desempeño docente en los niveles de educación inicial y primaria de la institución educativa, podemos señalar que ha sido probada ya que, según los resultados, existe una relación significativa entre cada uno de los estilos de liderazgo directivo y el desempeño docente, predominando el Liderazgo Transformacional.

Estos hallazgos coinciden con los de Huari (2007), quien reportó que los directores con un estilo de liderazgo facilitador, transformacional y controlador tienen una gestión buena 0 excelente. Cabe resaltar la importancia e influencia que el estilo de liderazgo del directivo puede ejercer sobre la gestión institucional, dentro del cual existen factores como clima institucional, relaciones interpersonales, remuneraciones, capacitaciones, incentivos, entre otros, que influyen en el desempeño docente.

Consideramos que el liderazgo directivo transformacional y estratégico tiene alguna característica en común, como el hecho de que el líder tiene clara la misión y visión de la organización e inspira a los docentes a tener una visión compartida. Es decir, a pesar de que el liderazgo directivo estratégico no es el estilo predominante en la institución educativa en estudio, se puede considerar que tiene una influencia significativa positiva en el desempeño docente pues comparte características del liderazgo transformacional.

En los resultados de la presente investigación, los docentes también perciben un estilo de Liderazgo Democrático, pero en un porcentaje menor.

Al parecer, el director de esta institución educativa promueve la participación del grupo sin dejar de tomar decisiones cuando sea necesario, permite las sugerencias para modificar las propuestas, es un líder que escucha, estimula y facilita el apoyo a la labor docente. Como parte de su organización, cuenta con subdirectores y coordinadores que están en permanente comunicación con los docentes y demás trabajadores. Se sabe que el director mantiene reuniones frecuentes con todo el equipo directivo, quienes llevan las opiniones y propuestas de los docentes para ser considerados en la toma de decisiones. 
Estos datos coinciden con los resultados encontrados por Sorados (2010) quien encontró que cuando las decisiones son tomadas democrática y oportunamente por los directivos, ello tiene incidencia en la calidad de la gestión de las instituciones educativas.

Con respecto a la hipótesis que señala que existe una relación directa y significativa entre los estilos de liderazgo directivo y el desempeño docente en el nivel de educación secundaria de la institución educativa, podemos señalar que no se probó la hipótesis; ningún estilo de liderazgo directivo se relaciona con la variable desempeño docente, ya que las correlaciones no son significativas.

Podemos indicar que existe una relación fuerte entre el liderazgo del director y el desempeño docente como indicador de la gestión directiva. Esto podemos verlo reflejado en las evaluaciones de los docentes, en su desempeño, donde se refleja un nivel de desempeño alto. Esto prueba que un adecuado estilo de liderazgo puede incrementar el desempeño de los agentes educativos en las instituciones educativas. Al respecto, como lo señalan Paz y Zamora (2014) un estilo de liderazgo directivo adecuado repercute en la gestión, existiendo una relación positiva y fuerte entre el estilo de liderazgo directivo y el desempeño docente, lo cual es un indicador de la gestión del director.

Es importante considerar que según los resultados obtenidos en esta investigación sí existe una relación directa y significativa entre los estilos de liderazgo directivo y el desempeño docente en la institución educativa, como también lo sustenta Calla (2008), en donde obtuvo como resultado la determinación de un alto grado de correlación entre el desempeño de los docentes y el estilo de liderazgo ejercido por los directores en su investigación. Como también en su tesis doctoral, Thieme (2006) sostiene en su investigación lo contrario: que no hay una asociación estadísticamente significativa si se compara la eficiencia técnica de la organización con el liderazgo ejercido.

También se puede observar en el presente estudio que las percepciones de los docentes son variadas, percibiendo en su mayoría que sus líderes son transformacionales y estratégicos y en un porcentaje menor democráticos y autocráticos. Estos estilos de liderazgo pueden influir de manera positiva o negativa en el desempeño docente. No existen líneas de un liderazgo definido; es decir, que un líder no tiene un estilo de liderazgo definido para conducir a un grupo, el líder puede manejar varios estilos dependiendo de las circunstancias donde tenga que ejercer su liderazgo y su personalidad. Sin embargo, habrá una tendencia hacia un estilo particular que lo caracterizará en la organización, debido a las cualidades que reúne y al cargo que desempeña. El directivo debe conocer su estilo de liderazgo, diagnosticar la situación particular en la que se encuentra y después buscar la coincidencia entre su estilo y la situación específica (Stoner, 1989). 


\section{CONCLUSIONES}

- Existe una relación directa y significativa entre los estilos de liderazgo directivo y el desempeño docente en la Institución Educativa Parroquial del distrito de San Isidro.

- Existen indicadores suficientes que muestran que el estilo de liderazgo que predomina en la Institución Educativa Parroquial del distrito de San Isidro, es el estilo de Liderazgo Transformacional.

- Los docentes presentan altos niveles de desempeño docente, debido a la buena gestión realizada por el directivo y por el estilo de liderazgo predominante en la institución educativa.

- Existen una relación directa y significativa entre los estilos de liderazgo directivo y el desempeño docente en el nivel de educación inicial y primaria en la Institución Educativa Parroquial del distrito de San Isidro, predominando el estilo Transformacional.

- No existen una relación directa y significativa entre los estilos de liderazgo directivo y el desempeño docente en el nivel de educación secundaria en la Institución Educativa Parroquial del distrito de San Isidro, ya que las correlaciones no son significativas.

\section{RECOMENDACIONES}

- Implementar dentro de las instituciones educativas talleres de liderazgo transformacional dirigido a docentes y directivos con el propósito de tener directores y maestros que sean agentes de cambios, comprometidos con su institución.

- Promover más investigaciones a fin de establecer diagnósticos de gestión de directores y en su capacitación en temas de liderazgo.

- Promover la identificación de instituciones educativas que cuenten con directores líderes transformacionales a fin de que sirvan de modelos a otros directores para la mejora de su gestión.

- Dar a conocer los resultados de la presente investigación a los directivos y docentes de la institución, para elaborar un plan de mejora, con la finalidad de optimizar los niveles de desempeño docente. 


\section{REFERENCIAS}

Alfonso, A. (2001). La excelencia gerencial en educación. Lima: Magisterial. Biblioteca Pública de Lima.

Álvarez, M. (1996). Manual de organización de instituciones educativas. Madrid: Escuela española.

Álvarez, M. (1998). El liderazgo de la calidad total. Madrid: Escuela española.

Ball, S. (1989). La micropolítica de la escuela. Hacia una teoría de la organización escolar. México: Paidós.

Bardisa, T. (1997). Teoría y práctica de la micropolítica en las organizaciones escolares. Revista Iberoamericana de Educación. N015. Organización de Estados Iberoamericanos para la educación, ciencia y la cultura. http://www.rieoei.org/oeivirt/rie15a01.htm

Bass, R. (1988). La gestión educativa ante la innovación y el cambio. Madrid: Narcea.

Beare, H., Caldwell, B. y Millikan, R. (1992). Cómo conseguir centros de calidad. Nuevas técnicas de dirección. Madrid: La Muralla.

Calla, C. (2008). El estilo de liderazgo de los directivos y su relación con el desempeño docente en la región del Callao-Lima. (Tesis de maestría). Universidad Enrique Guzmán y Valle, La Molina, Lima.

Capella, J. (2000). Planificación de la educación. Documento de Trabajo. Lima: PUCP.

Chiavenato, I. (2006). Administración en los nuevos tiempos. Bogotá- Colombia: Mc Graw Hill.

Chiavenato, I. (2009). Administración. Proceso administrativo. México: Mc Graw Hill.

Chiavenato, I. (2009). Gestión del talento humano. México: Mc Graw Hill.

Chiroque, S. (2006). Evaluación de desempeños docentes.

Recuperado de:http://www.educared.edu.pe/directivos/articulo/823/evaluacion-dedesempenos docentes/

Congreso Internacional sobre dirección de centros educativos (2000). Liderazgo y organizaciones que aprenden. Bilbao: Universidad de Deusto.

Crozier, M. y Friedberg, E. (1990). El actor y el sistema: las restricciones de la acción colectiva. México: Alianza Editorial. 
Gorrochotegui, A. (2007). Modelo para las enseñanzas de las competencias del liderazgo. Universidad de La Sabana, Facultad de Educación.

Hernández, R; Fernández, C. \& Baptista, P. (2014). Metodología de la Investigación. México: Ed. Mc. Graw Hill.

Huari, J. (2007). Estilos de liderazgo y la gestión institucional de los centros educativos nacionales y particulares del distrito y provincia del Callao. (Tesis de maestría) Universidad Enrique Guzmán y Valle, la Molina, Lima.

Ibáñez, M. (1996). Calidad total. Lima: Fondo Editorial de la Universidad Nacional Mayor de San Marcos.

Kernberg, O. (1999). Ideología, conflicto y liderazgo en grupos y organizaciones. Barcelona: Editores Paidós.

Kline, P. (1999). The handbook of psychological testing (2 ed.). Londres: Routledge.

Leithwood, K. (1994). Liderazgo para la reestructuración de las escuelas. Revista de educación 304.

Leithwood, K; Tomlinson, D. y Genge, M. (1996). Transformational School Leadership. Dordrecht/Boston/London: Kluwer Academic Publishers. International Handbook of Educational Leadership and Administration. Volumen 2.

Lussier, R. y Achua, C. (2004). Liderazgo: Teoría, Aplicación y desarrollo de habilidades. México. Thomson Editores.

Madrigal, B. (2005). Liderazgo. Enseñanza y Aprendizaje. México: McGraw-Hill Interamericana.

Madrigal, B. (2011). Liderazgo y aprendizaje. México: Mc Graw-Hill Interamericana Editores.

Maureira, O. (2004). El liderazgo factor de eficacia escolar, hacia un modelo causal. REICE. Revista Electrónica Iberoamericana sobre Calidad, Eficacia y Cambio en Educación. Recuperado de http://redalyc.uaemex.mx/pdf/551/55120108.pdf

Medina, P. (2010). El liderazgo transformacional en los docentes de un colegio de gestión cooperativa en la ciudad de Lima. (Tesis de maestría). Universidad Católica del Perú. Recuperado de http://blog.pucp.edu.pe/media/avatar777.doc 
Ministerio de Educación. Chile (2007). Evaluación de Desempeño. Manual para la elaboración y evaluación de compromisos. Unidad de gestión y mejoramiento educativo. Chile.

Mulford, B. (2006). Leadership for improving the quality of secondary education some international developments. Facultad de Educación, University of Tasmania . http:// www.urg.es/local/recfpro/Rev101ART.pdf

Orellana, 0. (2003). Enseñanza y Aprendizaje. Lima: San Marcos. 1era edición.

Paz, H. \& Zamora C. (2014). Estilos de liderazgo del directivo y su relación con la satisfacción laboral docente en dos instituciones educativas de Lima Metropolitana. (Tesis de maestría) Universidad Marcelino Champagnat, Surco, Lima.

Robbins, S. \& Jugde, T. (1999). Comportamiento organizacional. Naucalpan de Juárez: México. Pearson educación.

Romero, J. (2008). El desempeño docente como factor fundamental para la Calidad Educativa de la Educación Básica Venezolana.

Recuperado de: http://www.buenastareas.com/ensayos/El-Desempe\%C3\%B10Docente-Como-Factor Fundamental/452064.html

Sandoval, E. (2000). La trama de la escuela secundaria: Institución, relaciones y saberes. México. UPN/Plaza y Valdez.

Santos, M. (1997). La luz del prisma: para comprender las organizaciones educativas. Málaga: Ed. Aljibe.

Saravia, L. (2005). El desempeño docente: clave en la Carrera Pública Magisterial. Recuperado de: http://saravia.wordpress.com/2009/05/07/el-desempenodocente- $\neg$ claveen-la-carrera-publica-magisterial/

Saravia, L. \& López, M. (2008). La evaluación del desempeño docente. Perú, una experiencia en construcción. Revista Iberoamericana de evaluación educativa. Recuperado de http://www.rinace.net/riee/numeros/vol1-num2/art5.pdf

Sorados, M. (2010). Influencia del liderazgo en la calidad de la gestión educativa. (Tesis de maestría, universidad Nacional mayor de San Marcos). Recuperado de: http:// www.cybertesis.edu.pe/sisbib/2010/sorados_pm/pdf/sorados m.pdf 
Stoner, J. (1989). Administración. 3ra. Edición, Editorial Prentice Hall Hispanoamericana México.

Thieme, C. (2006). Liderazgo y eficiencia en la educación primaria. Barcelona (España): Universidad Autónoma de Barcelona, tesis doctoral.

Trechera, L. (2004). Habilidades de dirección, factores determinantes para desarrollar el liderazgo. Bilbao: Desclée de Brouwer.

UNESCO. (2004). Financiamiento y gestión de la educación en América Latina y el Caribe. San Juan de Puerto Rico: CEPAL.

Whetten, D. y Cameron, K. (2005). Management Developing Skills. México, D.F: Pearson Education.

Zela, M. (2008). Liderazgo y calidad educativa en las instituciones educativas secundarias en la provincia de Lampa - 2007. Juliaca (Perú). Universidad Alas Peruanas. Tesis de maestría. 